Received: 7 February 2019

Accepted: 7 May 2019

Published online: 20 May 2019

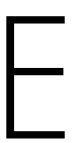

C
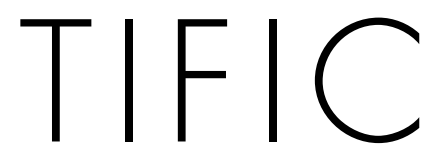

REP

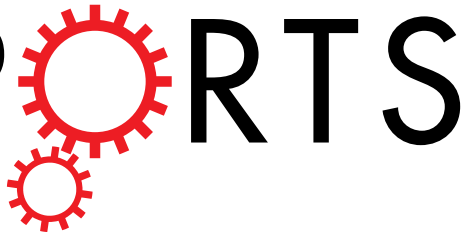

\title{
Covalent-bonding-induced strong phonon scattering in the atomically thin $\mathrm{WSe}_{2}$ layer
}

Young-Gwan Choi ${ }^{1,3}{ }^{3}$, Do-Gyeom Jeong ${ }^{1}$, H. I. Ju ${ }^{1}$, C. J. Roh ${ }^{1}$, Geonhwa Kim ${ }^{1}$, Bongjin Simon Mun ${ }^{1}$, Tae Yun Kim², Sang-Woo Kim $\mathbb{D}^{2}$ \& J. S. Lee ${ }^{1}$

In nano-device applications using two-dimensional (2D) van der Waals materials, a heat dissipation through nano-scale interfaces can be a critical issue for optimizing device performances. By using a time-domain thermoreflectance measurement technique, we examine a cross-plane thermal transport through mono-layered $(n=1)$ and bi-layered $(n=2) \mathrm{WSe}_{2}$ flakes which are sandwiched by top metal layers of $\mathrm{Al}, \mathrm{Au}$, and $\mathrm{Ti}$ and the bottom $\mathrm{Al}_{2} \mathrm{O}_{3}$ substrate. In these nanoscale structures with hetero- and homo-junctions, we observe that the thermal boundary resistance (TBR) is significantly enhanced as the number of $\mathrm{WSe}_{2}$ layers increases. In particular, as the metal is changed from $\mathrm{Al}$, to $\mathrm{Au}$, and to Ti, we find an interesting trend of TBR depending on the WSe $\mathrm{C}_{2}$ thickness; when referenced to TBR for a system without WSe $\mathrm{S}_{2}$ TBR for $n=1$ decreases, but that for $n=2$ increases. This result clearly demonstrates that the stronger bonding for Ti leads to a better thermal conduction between the metal and the WSe $\mathrm{layer}_{\text {, }}$ but in return gives rise to a large mismatch in the phonon density of states between the first and second $\mathrm{WSe}_{2}$ layers so that the $\mathrm{WSe}_{2}-\mathrm{WSe}_{2}$ interface becomes a major thermal resistance for $n=2$. By using photoemission spectroscopy and optical second harmonic generation technique, we confirm that the metallization induces a change in the valence state of $\mathrm{W}$-ions, and also recovers a non-centrosymmetry for the bi-layered $\mathrm{WSe}_{2}$.

With an advent of exfoliation technique for van der Waals (vdW) materials, there have been tremendous progresses in 2D nanotechnology using graphene, transition metal dichalcogenides (TMDs), and so on ${ }^{1-4}$. Novel functionality can be realized in artificial heterostructures prepared as a multi-stacking of different layers exploiting a relatively weak vdW bonding strength ${ }^{5-7}$. Since such artificial structures can be made ultimately in a form of ultrathin atomic layers, one of the key issues in those devices should be a heat management ${ }^{8-10}$. The cross-plane thermal resistance in ultrathin 2D materials should be contributed to mainly by the boundary (Kapitza) resistance due to their infinitesimal thickness. In understanding the heat dissipation through 2D materials, there have been several previous works about the nano-scale heat transport based on the phonon transmission spectrum across the interfaces. Chen et al. used a non-equilibrium molecular dynamics (NEMD) simulations and atomistic Green's function (AGF) approach to clarify the thermal transport in the metal/graphene/metal geometry ${ }^{11}$. They argued that depending on a metal-graphene bonding strength, a significant mismatch in the phonon density of states (pDOS) can occur between two adjacent graphene layers, and the graphene interlayer can be a major resistance source. In addition, Yan et al. focused on the thermal transport through the metal $/ \mathrm{MoS}_{2} / \mathrm{metal}_{\text {junc- }}$ tion with an AGF method ${ }^{12}$. They found that a strong bonding between the metal and TMD leads to an electron injection from metal to TMD, and the cross-plane thermal transport can be modulated as a result of the change in the pDOS. Ong et al. demonstrated that an encasement of graphene with a top $\mathrm{SiO}_{2}$ layer introduces a new heat transport channel for the low frequency phonons, and hence the graphene-substrate Kapitza resistance can be significantly reduced ${ }^{13}$.

Besides such theoretical works, there have been a few other experimental studies on the heat transport through the van der Waals materials, such as graphene and $\mathrm{MoS}_{2}{ }^{14-16}$. Nevertheless, much less experimental reports have been made yet particularly about the heat transport through the interface between metal-vdW materials. In this work, we investigate the thermal transport through ultrathin $\mathrm{WSe}_{2}$ layers in contact with metal layers of $\mathrm{Al}, \mathrm{Au}$,

${ }^{1}$ Department of Physics and Photon Science, School of Physics and Chemistry, Gwangju Institute of Science and Technology, Gwangju, 61005, South Korea. ${ }^{2}$ School of Materials Science and Engineering, Sungkyunkwan University, Suwon, 16419, South Korea. ${ }^{3}$ Present address: Department of Energy Science, Sungkyunkwan University, Suwon, 16419, South Korea. Correspondence and requests for materials should be addressed to J.S.L. (email: jsl@gist.ac.kr) 
(a)

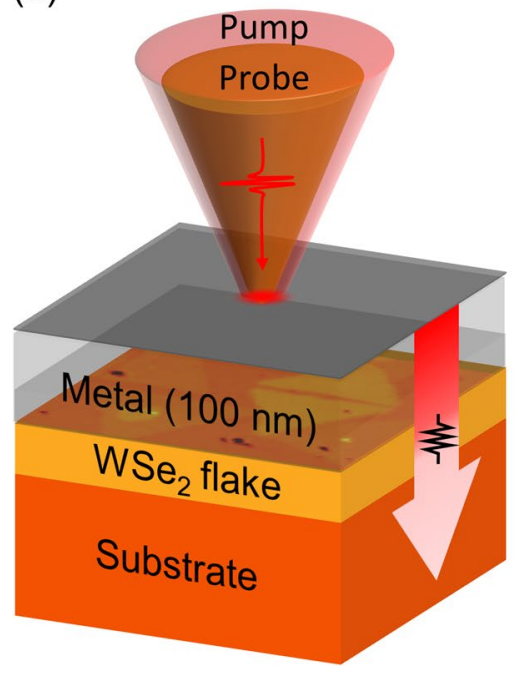

(b)

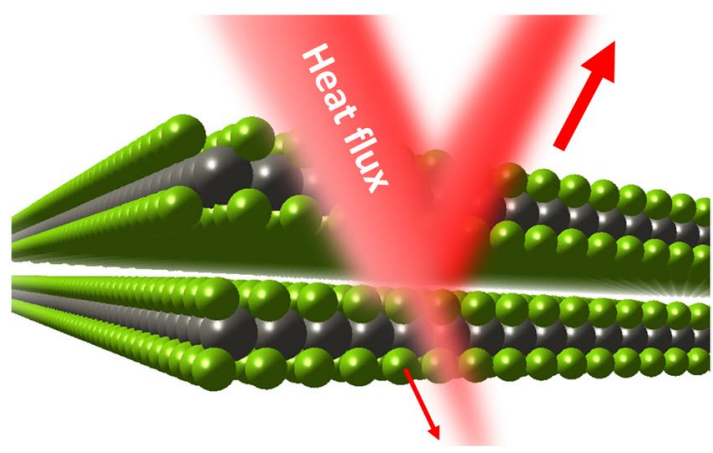

Figure 1. Schematic pictures of (a) the sample part in time-domain thermoreflectance experiment and (b) a cross-sectional view of a heat flux through WSe $e_{2}$ layers. In (a), an optical pump beam heats the metal surface, and the heat becomes dissipated through WSe ${ }_{2}$ flakes and finally to the substrate. Probe beam monitors a temperature change of the metal surface in terms of the reflectivity change. Figure (b) highlights a large thermal resistance of the $\mathrm{WSe}_{2}-\mathrm{WSe}_{2}$ interface which is due to a significant mismatch in the phonon density of states of neighboring $\mathrm{WSe}_{2}$ layers.

and Ti by using time-domain thermoreflectance (TDTR) technique which, as a pump-probe optical technique (Fig. 1(a)), provides us with key information about the cross-plane thermal boundary resistance. In particular, we focus on the role of metal contacts for minimizing the heat barrier in the $2 \mathrm{D}$ devices using vdW materials. Whereas all three metal layers lead to a change in the electronic structure of WSe $\mathrm{W}_{2}$ to have a finite density of states at the Fermi level, such tendency becomes stronger in the order of $\mathrm{Al}, \mathrm{Au}$, and $\mathrm{Ti}^{17,18}$. Therefore, we expect to learn how the electronic bonding strength between metal and $\mathrm{WSe}_{2}$ layers influences on the phonon transport through ultrathin vdW materials. Using the optical second harmonic generation technique, we found a clear signature of a non-centrosymmetric crystal structure of otherwise centrosymmetric bi-layer $\mathrm{WSe}_{2}$ due to the metallization of the top $\mathrm{WSe}_{2}$ layer. Also, we observed a corresponding change in the valence state of $\mathrm{W}$-ions using $\mathrm{x}$-ray photoemission spectroscopy. In the case of the thermal transport behavior, we observed a large difference of the thermal boundary resistance depending on the metal film. In particular, we could demonstrate that when WSe is more strongly bonded to the metal, e.g., Ti, the thermal transport through the metal-WSe interface becomes better, but in return it becomes worse for the $\mathrm{WSe}_{2}-\mathrm{WSe}_{2}$ interface, as schematically shown in Fig. 1(b). To minimize the thermal resistance in the $2 \mathrm{D}$ devices using $\mathrm{vdW}$ materials, it is therefore required to consider not only the hetero-interface formed between the metal and vdW layer, but also the homo-interface formed between two adjacent vdW layers.

\section{Distinct Thermal Properties of the Mono- and Bi-layered $\mathrm{WSe}_{2}$}

We prepared mono- and bi-layer WSe $e_{2}$ flakes, termed $n=1$ and $n=2$, respectively, on an $\mathrm{Al}_{2} \mathrm{O}_{3}(001)$ substrate. Optical images of the samples, shown in Fig. $2(\mathrm{a}-\mathrm{c})$, indicate that each flake has a triangular shape with a lateral size of about $10-20 \mu \mathrm{m}$. Mono- and bi-layered flakes are clearly distinguished from the color contrast; the brighter (darker) regions correspond to the bi-layer (mono-layer) flakes. To examine how the thermal transport

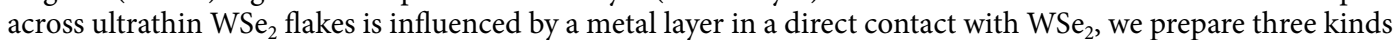
of metal (M), i.e., $\mathrm{Al}, \mathrm{Au}$, and Ti. Thickness of the metal layer is $100 \mathrm{~nm}$ for $\mathrm{Al}, 20 \mathrm{~nm}$ for $\mathrm{Au}$, and $2 \mathrm{~nm}$ for Ti. An additional $100 \mathrm{~nm}$ thick $\mathrm{Al}$ layer is prepared on top of $\mathrm{Au}$ and Ti. Although the Ti layer is relatively thinner than the other metal layers, its electrons state should be comparable to that of the thicker, e.g., $10 \mathrm{~nm}$, Ti film ${ }^{19}$ so that its covalent bonding ability with the WSe $\mathrm{W}_{2}$ layer is guaranteed.

We first examine a spatial distribution of the cross-plane thermal property by mapping a TDTR signal. (Refer to the experimental section for the details of the measurements.) Figure 2(d-f) display spatial mapping results of the $-\mathrm{V}_{\text {in }} / \mathrm{V}_{\text {out }}$ signal for $\mathrm{M}=\mathrm{Al}, \mathrm{Au}$, and Ti, respectively, where the signal at each point is taken with a $5 \mu \mathrm{m}$ spatial resolution and a $1 \mu \mathrm{m}$ interval. Ratio value, $-\mathrm{V}_{\text {in }} / \mathrm{V}_{\text {out }}$, is picked up at $1 \mathrm{~ns}$ of the time delay, and it is roughly proportional to thermal boundary conductance. In this TDTR mapping data, mono- and bi-flakes are clearly distinguished as in the optical images taken for the same area (see details in Supplementary Information). This suggests that the thermal boundary resistance should be given differently for $n=1$ and $n=2$ although their thicknesses are different only by about $0.6 \mathrm{~nm}$.

Furthermore, it is worthwhile to note that the color contrast between $n=1$ and $n=2$ is the largest for the Ti contact. Kang et al. and Giovannetti et al. suggested that TMDs and graphene can be metallized due to the covalent bonding or the chemical potential difference with the metal layer ${ }^{18,20}$. Moreover, Chen et al. and Yan et al. suggested that these charge redistributions can perturb the pDOS of graphene and TMDs ${ }^{11,12}$. In this respect, 
(a)

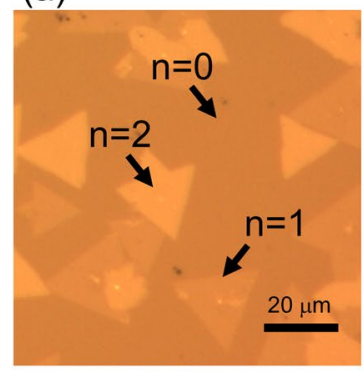

(b)

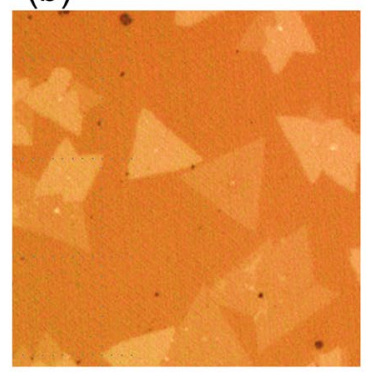

(c)

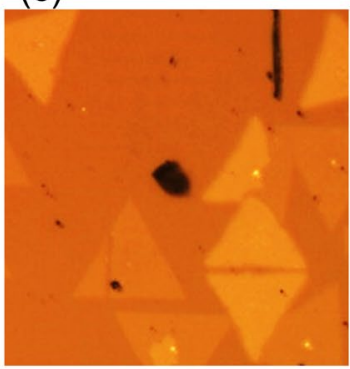

(d)

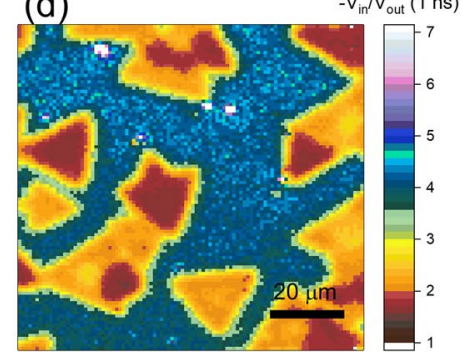

(e)

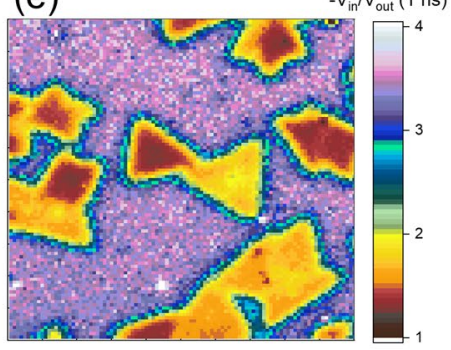

(f)

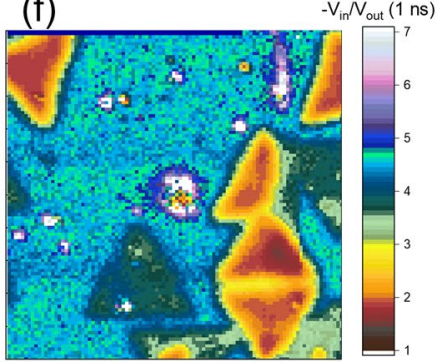

Figure 2. (a-c) Optical images of $\mathrm{WSe}_{2}$ flakes prepared on an $\mathrm{Al}_{2} \mathrm{O}_{3}$ substrate. (d-f) Time-domain thermoreflectance mapping results for $\mathrm{Al} / \mathrm{WSe} \mathrm{Al}_{2} / \mathrm{Al}_{2} \mathrm{O}_{3}, \mathrm{Al} / \mathrm{Au} / \mathrm{WSe}_{2} / \mathrm{Al}_{2} \mathrm{O}_{3}$, and $\mathrm{Al} / \mathrm{Ti} / \mathrm{WSe} \mathrm{C}_{2} / \mathrm{Al}_{2} \mathrm{O}_{3}$. The ratio signal $-\mathrm{V}_{\text {in }} / \mathrm{V}_{\text {out }}$ is monitored at the pump-probe time delay fixed at $1 \mathrm{~ns}$. In both images, mono- and bi-flakes have distinctive contrasts.

the charge redistributions in the upper WSe $\mathrm{W}_{2}$ layer will have a strong influence on the thermal transport in the metal-TMD-insulator heterostructure, and such effect should appear differently depending on the bonding strength of the metal layer in contact with TMD materials.

\section{Modulation of the Thermal Boundary Resistance by a Metal-WSe ${ }_{2}$ Bonding}

For a more quantitative analysis of the thermal boundary resistance, we obtained a full decay profile of $-\mathrm{V}_{\text {in }} / \mathrm{V}_{\text {out }}$ signals for each region of the samples, i.e., $n=1$ and $n=2$ as well as $n=0$ where the metal has a direct contact with the $\mathrm{Al}_{2} \mathrm{O}_{3}$ substrate. As displayed in Fig. 3(a-c), the three areas exhibit distinct time-dependent behaviors of the signal, and the relative values of each region are given consistently with the mapping results shown in Fig. 2. By considering the heat diffusion equation, we could model these time-dependent signals; the entire system is assumed to be composed of two layers of the metal transducer and the sapphire substrate, and their interface in between. In particular, the mono- and bi-flakes are so thin that their contribution to the thermal transport is taken into account as a part of the thermal boundary resistance. Each flake was measured three times at different positions, and 20 different flakes were examined in total. An average of all these results are taken with their standard deviations as error bars. The thermal conductivity of the sapphire is determined as $28.7 \pm 2.5 \mathrm{~W} / \mathrm{mK}$. Details of the modelling and analysis can be found elsewhere ${ }^{21}$.

Figure $3(\mathrm{~d}-\mathrm{f})$ display the obtained thermal boundary resistance (TBR) $\mathrm{R}(=1 / \mathrm{G})$ of $n=0,1$, and 2 for $\mathrm{M}=\mathrm{Al}$, $\mathrm{Au}$, and $\mathrm{Ti}$, respectively. In this work, the TBR is defined as $\mathrm{R}=\left(\mathrm{T}_{2}-\mathrm{T}_{1}\right) / \mathrm{F}$, where $\mathrm{F}$ is heat flux, $\mathrm{T}_{i}$ is temperature in a medium $i$ with $i=1$ and 2 for the metal and WSe ${ }_{2} / \mathrm{Al}_{2} \mathrm{O}_{3}$ layers, respectively. For $n=0$, TBR at the metal-substrate interface is in the range of $\sim 1-2 \times 10^{-8} \mathrm{~m}^{2} \mathrm{~K} / \mathrm{W}$. Although TBR can be varied depending on a surface condition and an evaporation technique, these values are in good agreement with previous reports of the TBR for the similar interfaces, such as $2.2 \times 10^{-8} \mathrm{~m}^{2} \mathrm{~K} / \mathrm{W}$ for $\mathrm{Au} / \mathrm{Al}_{2} \mathrm{O}_{3}$ and $0.95 \times 10^{-8} \mathrm{~m}^{2} \mathrm{~K} / \mathrm{W}$ for $\mathrm{Al} / \mathrm{Al}_{2} \mathrm{O}_{3}{ }^{22}$. For all metal contacts, there is a large increase of $\mathrm{R}$ by about $2-6 \times 10^{-8} \mathrm{~m}^{2} \mathrm{~K} / \mathrm{W}$ as the $\mathrm{WSe}_{2}$ flakes are inserted between the metal and the substrate. Note that a similar increase of TBR was experimentally observed when the graphene or $\mathrm{MoS}_{2}$ layer is inserted between $\mathrm{Au} / \mathrm{Ti}$ and $\mathrm{SiO}_{2}{ }^{14,15}$, or when the graphene is located between $\mathrm{Al}$ and $\mathrm{Cu}^{16}$. As the number of the $\mathrm{WSe}_{2}$ layer increases from $n=1$ to $n=2$, a further increase of $\mathrm{R}$ is observed. Such an increase of $\mathrm{R}$ is particularly large for the Ti contact.

To get a better insight into these behaviors of the thermal boundary resistance, we consider a bonding density at the metal-WSe $e_{2}$ junction. As the covalent bonding between the metal and $\mathrm{WSe}_{2}$ becomes stronger, the electron density at their interface becomes higher. Hence, we can consider this bonding density as a measure of the bonding strength between the metal and $\mathrm{WSe}_{2}$. We take the corresponding values from the previous theoretical works by Liu et al. and Kang et al., i.e., $0.008 / \AA^{3}$ for $\mathrm{Al}, 0.018 / \AA^{3}$ for $\mathrm{Au}$, and $0.029 / \AA^{3}$ for $\mathrm{Ti}^{17,18}$. Here, we introduce the ratio of thermal boundary conductance $\mathrm{G}(=1 / \mathrm{R})$ to denote a phonon transmittivity. Figure $4(\mathrm{a})$ shows that as the bonding density increases, $\mathrm{G}(n=1) / \mathrm{G}(n=0)$ increases, but $\mathrm{G}(n=2) / \mathrm{G}(n=1)$ seems to decrease.

Here, the relative thermal conductance between $n=1$ and $n=0$ is less than 0.4 for all three cases, and such a reduction in $\mathrm{G}(n=1)$ is attributed to the van der Waals bonding nature of the $\mathrm{WSe}_{2}$ layer or the interface roughness ${ }^{15}$. Nevertheless, the relative value of $\mathrm{G}(n=1)$ is the largest for the Ti contact where the bonding density is at the 

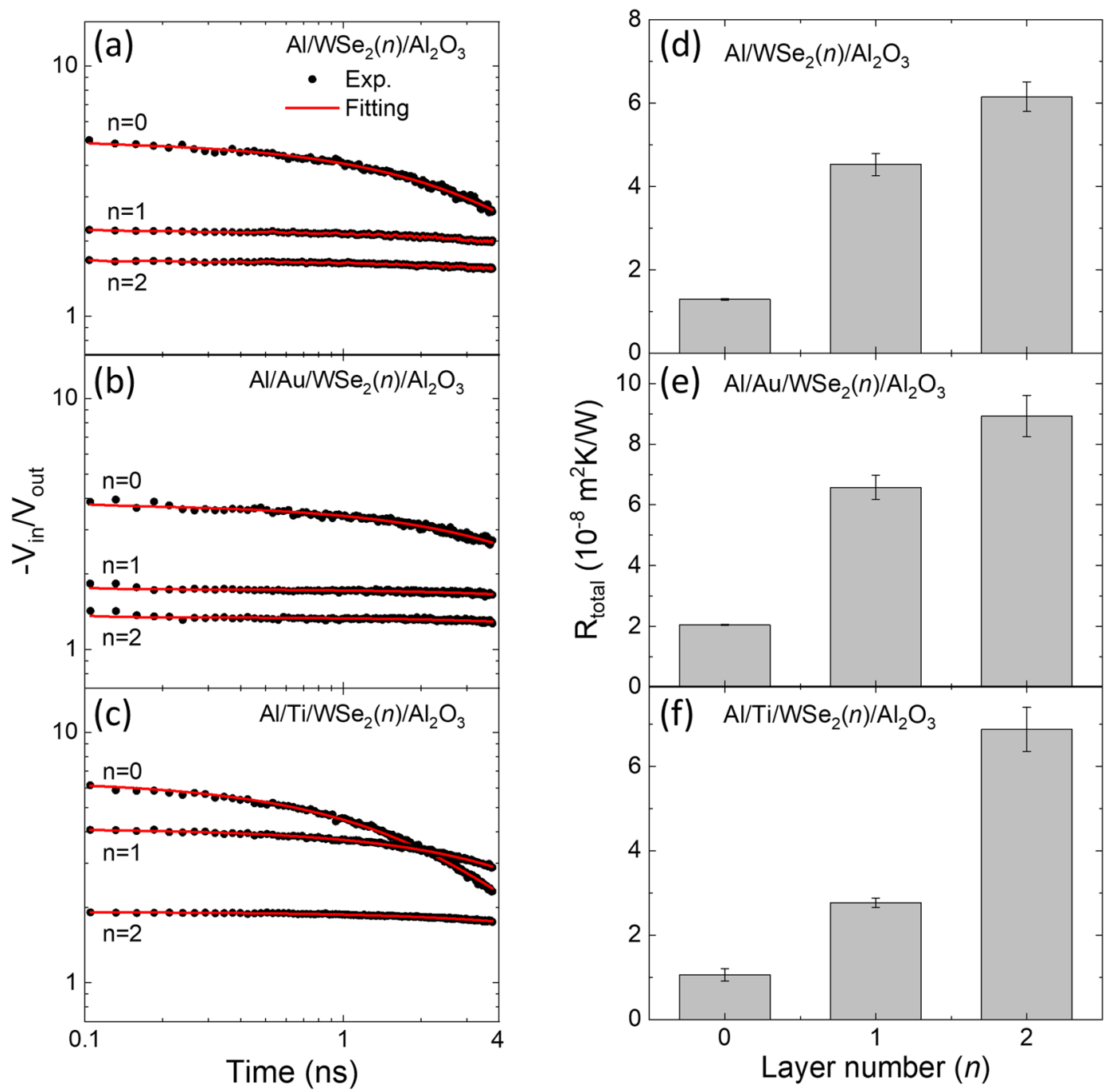

Figure 3. (a-c) Time-domain thermoreflectance data (symbol) for $\mathrm{Al} / \mathrm{WS} \mathrm{W}_{2} / \mathrm{Al}_{2} \mathrm{O}_{3}, \mathrm{Al} / \mathrm{Au} / \mathrm{WSe}_{2} / \mathrm{Al}_{2} \mathrm{O}_{3}$, and $\mathrm{Al} /$ $\mathrm{Ti} / \mathrm{WSe}_{2} / \mathrm{Al}_{2} \mathrm{O}_{3}$. In each case, the number of $\mathrm{WSe}_{2}$ layers is indicated by $n=0,1$, and 2 . The fitting curves (solid lines) for the time-domain data are also shown. $(\mathbf{d}-\mathbf{f})$ Extracted total thermal boundary resistance $\mathrm{R}_{\text {total }}$ values depending on the layer number $n$.
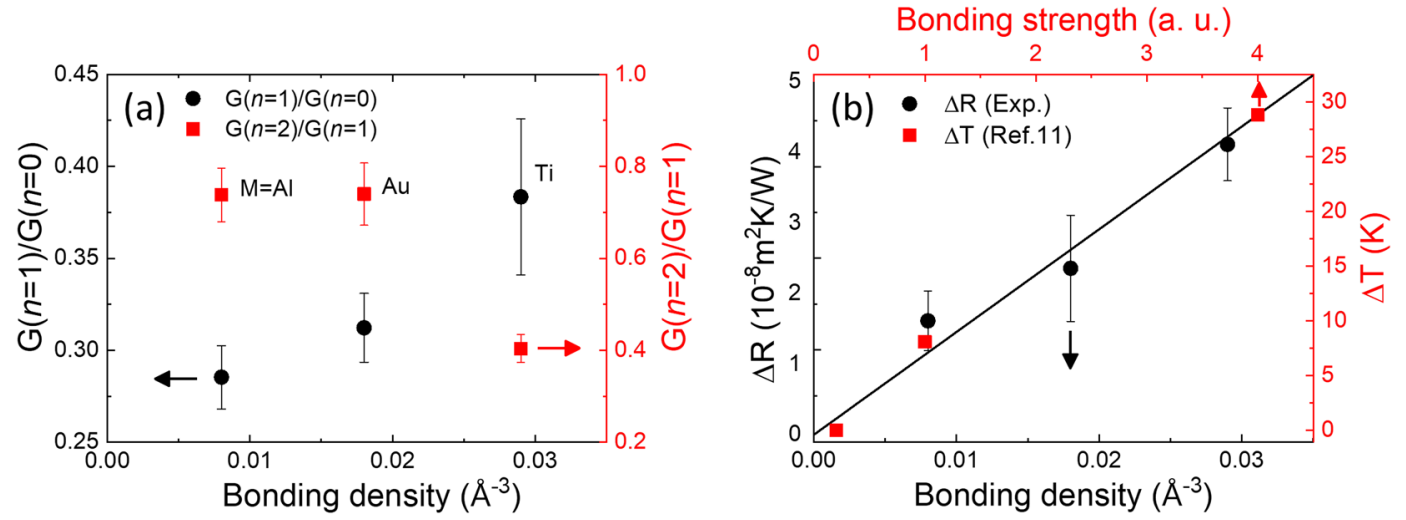

Figure 4. (a) Thermal boundary conductance ratio between $n=1$ and 0 (left axis), and between $n=2$ and 1 (right axis). The values are displayed as a function of the bonding electron density at the metal-WSe $\mathrm{H}_{2}$ interface which are quoted from refs $^{17,18}$. (b) Thermal boundary resistance difference between $n=1$ and 0 (black circle) and a temperature-drop in multi-layer graphene (red square) quoted from ref. ${ }^{11}$. 
maximum. Given the perfect crystalline quality of the interface, the thermal boundary resistance arises as a result of the scattering of the phonons which is due to the pDOS mismatch between the metal and WSe layer $^{14}$. As the metal-2D layer bonding becomes stronger, the pDOS of the 2D layer shifts toward that of the bonding material $^{11,12}$, and hence the pDOS mismatch can be reduced leading to the enhancement of $\mathrm{G}(n=1)$. In the same context, we can discuss the possible role of the $\mathrm{WSe}_{2} / \mathrm{WSe}_{2}$ interface in the cross-plane thermal transport by comparing $\mathrm{G}(n=2) / \mathrm{G}(n=1)$. Contrary to $\mathrm{G}(n=1) / \mathrm{G}(n=0), \mathrm{G}(n=2) / \mathrm{G}(n=1)$ exhibits the smallest value for the $\mathrm{Ti}$ contact with the largest bonding density. Although the strong chemical bonding between $\mathrm{Ti}$ and WSe $\mathrm{W}_{2}$ layers has a significant effect on the pDOS of WSe $e_{2}$, such effect would be limited only up to the first layer of WSe $\mathrm{C}_{2}$. This leads to the significant pDOS mismatch between upper and lower WSe $\mathrm{C}_{2}$ layers, and hence results in the stronger phonon scattering as schematically shown in Fig. 1(b) ${ }^{11}$.

To highlight the thermal boundary resistance occurring at the $\mathrm{WSe}_{2}-\mathrm{WSe}_{2}$ interface, we examine the additional resistance for $n=2$ compared to the case of $n=1$. Namely, we obtain $\Delta \mathrm{R}=\mathrm{R}(n=2)-\mathrm{R}(n=1)$, and find that it ranges from $1.5 \times 10^{-8} \mathrm{~m}^{2} \mathrm{~K} / \mathrm{W}$ for $\mathrm{Al}$ to $4 \times 10^{-8} \mathrm{~m}^{2} \mathrm{~K} / \mathrm{W}$ for Ti as shown in Fig. $4(\mathrm{~b})$. These values are unexpectedly large when they are considered as boundary resistances at the homo-junction formed by the same kind of materials. Actually, the metal/semiconductor heterojunctions have the TBR values in a range of $0.5-10 \times 10^{-8} \mathrm{~m}^{2} \mathrm{~K} / \mathrm{W}^{23}$, which can be confirmed also for $n=0$ as shown in Fig. 3(b). WSe $e_{2}$ double layers are successively deposited by using a chemical vapor deposition technique, and hence we can ignore the effect of interstitial voids, bubbles, or dirt that should be considered importantly in other sample preparation techniques, such as exfoliation and transfer method $\mathrm{s}^{24-26}$. Rather, since $\Delta \mathrm{R}$ increases linearly with an increase of the bonding density, we consider a strong hybridization between the metal and the first WSe $\mathrm{Wlyer}_{2}$ as a major reason of such a large $\Delta R$. Actually, similar behaviors can be found also in the graphene layers in contact with the metal. In the tri-layer graphene sandwiched by metal layers, Chen et al. showed using the NEMD simulations that the TBR at the graphene-graphene interface becomes larger as the metal-graphene bonding becomes stronger. We include such behavior with red squares in Fig. 4(b); a temperature-drop $(\Delta \mathrm{T})$ across the graphene-graphene interfaces increases in proportion to the bonding strength ${ }^{11}$. These results of $\mathrm{WSe}_{2}$ and graphene strongly suggest that the interface formed by two vdW materials could be a major thermal resistance in ultrathin devices made of vdW layers particularly in contact with metal layers. Similarly, for multi-layered $\mathrm{MoS}_{2}$, Goni et al. demonstrated that the thermal boundary conductance drops significantly from $n=1$ to $n=2$ in good agreement with our experimental results ${ }^{15}$. As $n$ increases further, the reduction of thermal boundary conductance becomes less significant since adjacent layers should have similar phonon density of states, which is expected also for our WSe ${ }_{2}$ system.

\section{Electronic and Crystal Structural Properties of Surface and Interface of WSe $\mathrm{S}_{2}$}

To confirm the bonding configuration between the metal and TMD materials, we conduct x-ray photo-emission spectroscopy (XPS) measurement (see details in Supplementary Information). Among three metal contacts, we check the Al case. Figure 5(a,b) displays W 4 f peaks obtained for a bare $\mathrm{WSe}_{2}$ and the $\mathrm{Al}(5 \mathrm{~nm}) / \mathrm{WSe}_{2}$ specimen. Two peaks around $32.2 \mathrm{eV}$ and $34.4 \mathrm{eV}$ correspond to the doublet $\mathrm{W} 4 \mathrm{f}_{7 / 2}$ and $\mathrm{W} 4 \mathrm{f}_{5 / 2}$, respectively ${ }^{27-29}$, of the $\mathrm{WSe}_{2}$ composition. The other two peaks around $35.8 \mathrm{eV}$ and $38.0 \mathrm{eV}$ correspond to the doublet $\mathrm{W} 6 \mathrm{f}_{7 / 2}$ and $\mathrm{W}$ $6 \mathrm{f}_{5 / 2}$, which are attributed to the $\mathrm{WO}_{3}$ precursor ${ }^{27}$. We assume that doublet $\mathrm{W} 6 \mathrm{f}_{7 / 2}$ and $\mathrm{W} 6 \mathrm{f}_{5 / 2}$ responses do not change depending on specimens due to the constant density of $\mathrm{WO}_{3}$ seeds. With this assumption, comparison between the two set of XPS responses reveals that the doublet $\mathrm{W} 4 \mathrm{f}_{7 / 2}$ and $4 \mathrm{f}_{5 / 2}$ responses are reduced when $\mathrm{WSe}_{2}$ is in contact with Al. Actually, this is attributed to the formation of $\mathrm{W}_{2} \mathrm{Se}_{3}$, which originates from the covalent bonding between $\mathrm{Al}$ and $\mathrm{WSe}_{2}$ layers ${ }^{27-29}$.

A similar bonding nature between the metal contact and $\mathrm{WSe}_{2}$ layer can be manifested by utilizing the optical second harmonic generation (SHG) measurement ${ }^{30}$. For the mono-layer $\mathrm{WSe}_{2}$, it shows a strong SHG signal due to the broken inversion symmetry; a three-fold rotational symmetry is clearly observed for the SHG signal obtained as a function of the sample azimuth in a normal-incidence reflection geometry. And, this anisotropy is in agreement with the $3 \mathrm{~m}$ point group ${ }^{31-33}$. For bi-layer TMDs, on the other hand, the centrosymmetry is usually

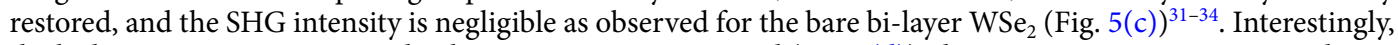
the bi-layer $\mathrm{WSe}_{2}$ in contact with Al gives a strong SHG signal (Fig. 5(d)); the SHG intensity amounts to almost half of the intensity observed for the mono-layer $\mathrm{WSe}_{2}$. This observation reflects a change in a structural symmetry, and can be understood in a similar way with the electronic state change observed by XPS; the metal-WSe $\mathrm{W}_{2}$ bonding induces a change in the structural property of the upper $\mathrm{WSe}_{2}$ layer, and its combination with the intact lower $\mathrm{WSe}_{2}$ layer leaves the bi-layer $\mathrm{WSe}_{2}$ to be inversion asymmetric ${ }^{31-34}$.

\section{Discussion}

Consequently, we can consider the changes in electronic state and crystal symmetry of the top WSe $e_{2}$ layers as one of the key factors in understanding the observed significant modulation of the thermal boundary resistance in the metal/WSe $e_{2}$ sapphire structure. Namely, the metallization of the $\mathrm{WSe}_{2}$ layer actually occurs by a strong bonding between metal and $\mathrm{WSe}_{2}$ which is expected to modify the phonon density of states in the $\mathrm{WSe}_{2}$ layer. Although this process would increase the phonon transmission across the metal-WSe $\mathrm{W}_{2}$ interface, it can have an opposite effect on otherwise the homo-interface between adjacent $\mathrm{WSe}_{2}$ layers. To realize $2 \mathrm{D}$ devices with an efficient heat dissipation property, the net thermal transport should be optimized by considering the heat dissipation efficiencies not only of hetero-junctions, e.g., the metal-WSe ${ }_{2}$ interface, but also of the homo-junctions, e.g., the $\mathrm{WSe}_{2}-\mathrm{WSe}_{2}$ interface.

Sample preparation and characterization. The monolayer and bilayer $\mathrm{WSe}_{2}$ were synthesized by chemical vapor deposition (CVD) method. CVD chamber is divided into two zones. The Se (0.2 g, 99.99\%) powders were put on an alumina boat placed in the middle of the first heating zone, and the $\mathrm{WO}_{3}$ powders $(0.01 \mathrm{~g}$, $99.995 \%)$ were put on an alumina boat placed in the middle of the second heating zone. The sapphire substrates 

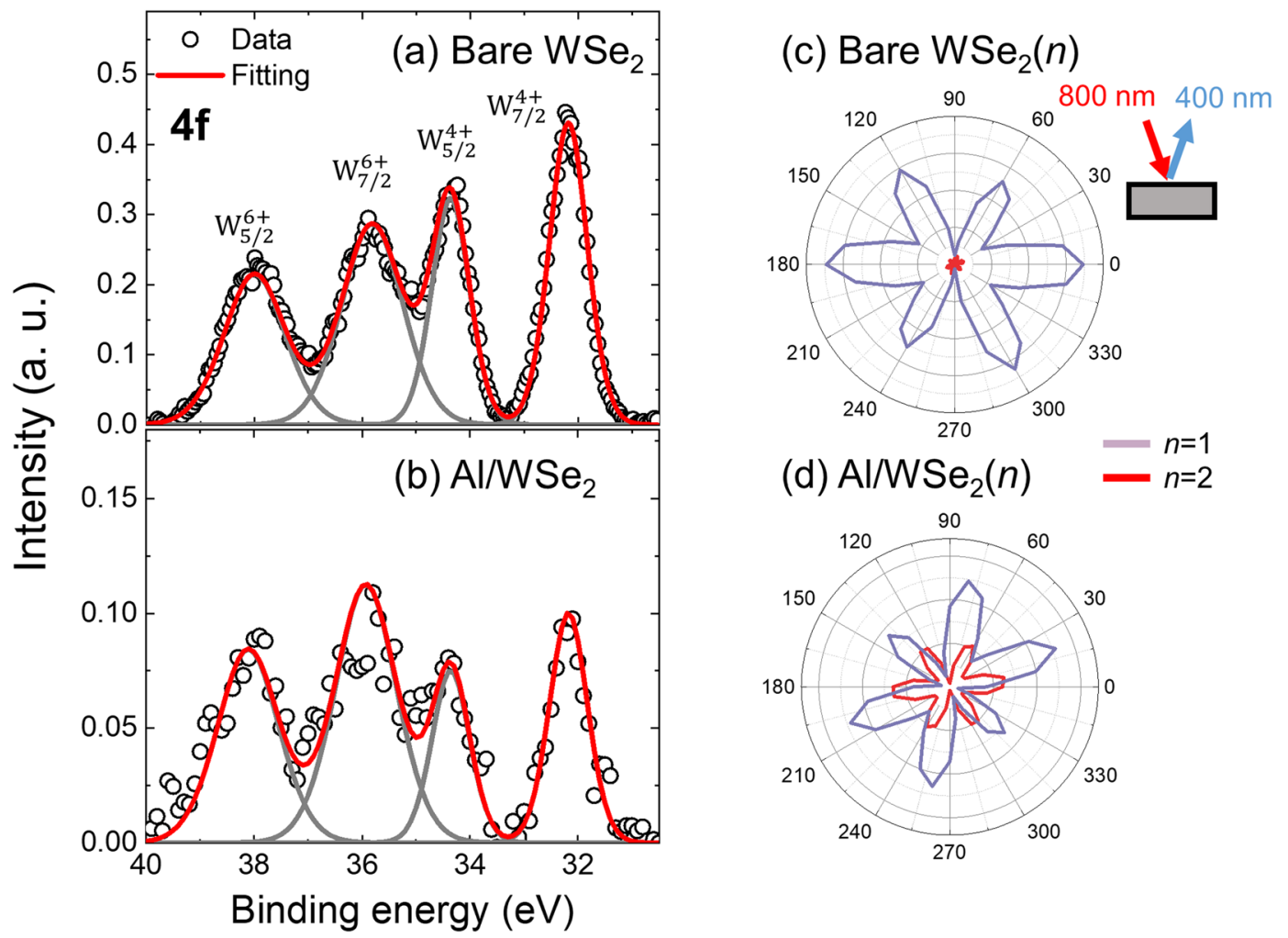

Figure 5. (a,b) X-ray photoemission spectroscopy data around the $\mathrm{W} 4 \mathrm{f}$ peaks for a bare $\mathrm{WSe}_{2}(\mathbf{a})$ and $\mathrm{Al} / \mathrm{WSe}_{2}$ (b). Four peaks can be fitted with contributions of $\mathrm{W}^{6+}$ and $\mathrm{W}^{4+}$ states, and two peaks of $\mathrm{W}^{4+}$ states become weaker for $\mathrm{Al} / \mathrm{WSe}_{2}$ sample. (c,d) Azimuth-dependent second-harmonic generation (SHG) intensity from $\mathrm{WSe}_{2}$ flakes with a layer number $n=1$ and 2. Inset shows a schematic of the SHG experiment where red and blue arrows indicate the fundamental $(800 \mathrm{~nm})$ and frequency-doubled $(400 \mathrm{~nm})$ waves, respectively. The SHG intensity for $n=2$ is ignorable for a bare WSe ${ }_{2}$, but it becomes almost half of the $n=1$ case for a WSe $\mathrm{W}_{2}$ with an $\mathrm{Al}$ layer on its top.

for deposition and growth of $\mathrm{WSe}_{2}$ were put at the right side of $\mathrm{WO}_{3}$ powders in the second heating zone. Argon gas for carrier gas and hydrogen for reductant were used with flow rate of $190 \mathrm{sccm}$ and $10 \mathrm{sccm}$, respectively. During the synthesis of monolayer (and bilayer) WSe $e_{2}$, first and second zones were heated up to $150{ }^{\circ} \mathrm{C}$ and $925^{\circ} \mathrm{C}$ (and $875^{\circ} \mathrm{C}$ for bilayer $\mathrm{WSe}_{2}$ ), respectively, for $37 \mathrm{~min}$ and then maintained for $30 \mathrm{~min}$. After finishing the growth, the chamber was naturally cooled down to room temperature. Using the multi-target e-beam evaporator, $100 \mathrm{~nm}$ thick $\mathrm{Al}$ is deposited for all three cases, whereas $\mathrm{Au}$ and $\mathrm{Ti}$ are pre-deposited onto a $\mathrm{WSe}_{2}$ specimen with a thickness $20 \mathrm{~nm}$ and $2 \mathrm{~nm}$, respectively. The thickness of the entire metal layer is measured by acoustic echo signals from TDTR measurements ${ }^{35-37}$.

Time-domain thermoreflectance (TDTR) measurement. We used TDTR to observe temporal temperature excursion and also to extract thermal parameters, such as the thermal conductivity and thermal boundary resistance ${ }^{14,21,36,37}$. As a light source for this pump-probe measurement, we used femtosecond laser pulses with a $785 \mathrm{~nm}$ wavelength and a repetition rate of $80 \mathrm{MHz}$. The beam was split for the pump and probe sources by a polarizing beam splitter, and the pump beam was modulated by an electro-optic modulator with a frequency $10 \mathrm{MHz}$. As schematically shown in Fig. 1(a), the pump-induced temperature change of the Al thermal transducer was monitored by the reflectivity change of the probe beam of which arrival time at the sample position was adjusted by a 300-mm-long delay stage. Both beams were focused by a $10 \times$ magnitude objective lens onto the sample where the beam size was about $5 \mu \mathrm{m}$ of $1 / \mathrm{e}^{2}$ diameter. Due to the substantially large beam size $(\sim 5$ $\mu \mathrm{m})$ compared with a radial heat diffusion length $(\sim 0.6 \mu \mathrm{m})$, we can selectively measure the heat transfer along the out-of-plane direction. Particularly for $\mathrm{WSe}_{2}$, the lateral heat transfer is negligible due to its small thickness of about $1 \mathrm{~nm}$. The beam size and its circular shape were maintained in an entire range of the time-delay ${ }^{38}$. To avoid an unnecessary detection of the pump beam, we separated pump and probe beams in a wavelength domain by adopting a two-tint method, and used appropriate optical filters: $785 \mathrm{~nm}$ long pass filter for the pump beam and $785 \mathrm{~nm}$ short pass filters for the probe beam ${ }^{39}$. A probe signal was detected by a photo-diode, and fed into a fast pre-amplifier and an electrical $10 \mathrm{MHz}$ band-pass filter. Finally, the signal was taken by using a radio frequency lock-in amplifier which provided in-phase $\left(\mathrm{V}_{\text {in }}\right)$ and out-of-phase $\left(\mathrm{V}_{\text {out }}\right)$ signals. We monitored their ratio, i.e., $-V_{\text {in }} / V_{\text {out }}$ with a variation of the sample position as well as the pump-probe delay time ${ }^{21}$. We also simulate 
sensitivity for TDTR analysis (see details in Supplementary Information), and confirm that the TDTR measurement has the high enough sensitivity to the thermal boundary conductance.

X-ray photoemission spectroscopy. The configuration of a chemical bonding between metal and TMD material was examined by utilizing x-ray photoemission spectroscopy. Entire measurements were carried out under an ultra-high vacuum condition at room temperature. The excitation source of $x$-ray was an aluminum $\mathrm{K}$-alpha with an energy $1486.6 \mathrm{eV}$, and a hemispherical electron analyzer from ScientaOminkron, Hipp-3 analyzer, was employed.

Optical second-harmonic generation. We employed an optical second-harmonic generation technique to examine a structural symmetry of the mono- and bi-layered WSe $\mathrm{W}_{2}$ flakes. We adopted a femtosecond laser with an $800 \mathrm{~nm}$ wavelength and an $80 \mathrm{MHz}$ repetition rate as a fundamental wave source, and focused it on the flakes with a beam size of $1 \mu \mathrm{m}$ in a normal incidence. The second harmonic wave was collected in a reflection geometry by using a photo-multiplier tube. Polarization states of the fundamental and the second harmonic waves were controlled by using a half wave plate and polarizer, respectively.

\section{References}

1. Novoselov, K. S. et al. Electric Field Effect in Atomically Thin Carbon Films. Science 306, 666-669 (2004)

2. Novoselov, K. S. et al. Two-dimensional atomic crystals. Proc. Natl. Acad. Sci. USA 102, 10451-10453 (2005).

3. Lin, Y. M. et al. 100-GHz Transistors from Wafer-Scale Epitaxial Graphene. Science 327, 662 (2010).

4. Chhowalla, M. et al. The chemistry of two-dimenional layered transition metal dichalcogenide nanosheets. Nat. Chem. 5, 263 (2013).

5. Koma, A. Van der Waals epitaxy for highly lattice-mismatched systems. J. Cryst. Growth 201-202, 236-241 (1999).

6. Lee, C.-H. et al. Atomically thin p-n junctions with van der Waals heterointerfaces. Nat. Nanotechnol. 9, 676 (2014).

7. Choi, W. et al. Recent development of two-dimensional transition metal dichalcogenides and their applications. Mater. Today 20, 116 (2017)

8. Pop, E., Sinha, S. \& Goodson, K. E. Heat Generation and Transport in Nanometer-Scale Transistors. Proc. IEEE 94, 1587 (2006)

9. Pop, E. Energy Dissipation and Transport in Nanoscale Devices. Nano Res. 3, 147-169 (2010).

10. Cahill, D. G. et al. Nanoscale thermal transport. II. 2003-2012. Appl. Phys. Rev. 1, 011305 (2014).

11. Chen, L., Huang, Z. \& Kumar, S. Impact of bonding at multi-layer grahene/metal interfaces on thermal boundary conductance. RSC adv. 4, 35852 (2014).

12. Yan, Z., Chen, L., Yoon, M. \& Kumar, S. The Role of Interfacial Electronic Properties on Phonon Transport in Two-Dimensional $\mathrm{MoS}_{2}$ on Metal Substrates. ACS Appl. Mater. Interfaces 8, 33299-33306 (2016).

13. Ong, Z.-Y., Cai, Y. \& Zhang, G. Theory of substrate-directed heat dissipation for single-layer graphene and other two-dimensional crystals. Phys. Rev. B 94, 165427 (2016).

14. Koh, Y. K., Bae, M.-H., Cahill, D. G. \& Pop, E. Heat Conduction across Monolayer and Few-Layer Graphenes. Nano Lett. 10, 4363-4368 (2010).

15. Goni, M., Yang, J. \& Schmidt, A. J. Enhanced thermal transport across monolayer $\mathrm{MoS}_{2}$. Nano Res. 11, 2173 (2018).

16. Kim, J. et al. Bimodal Control of Heat Transport at Graphene-Metal Interfaces Using Disorder in Graphene. Sci. Rep. 6, 34428 (2016).

17. Liu, W. et al. Role of Metal Contacts in Designing High-Performance Monolayer n-Type WSe ${ }_{2}$ Field Effect Transistors. Nano Lett. 13, 1983-1990 (2013).

18. Kang, J., Liu, W., Sarkar, D., Jena, D. \& Banerjee, K. Computational Study of Metal Contacts to Monolayer Transition-Metal Dichalcogenide. Semiconductors. Phys. Rev. X 4, 031005 (2014).

19. Güvenç, A. Effects of thickness on electronic structure of titanium thin films. Bull. Mater. Sci. 37, 41-45 (2014)

20. Giovannetti, G. et al. Doping graphene with metal contacts. Phys. Rev. Lett. 101, 026803 (2008).

21. Cahill, D. G. Analysis of heat flow in layered structures for time-domain thermoreflectance. Rev. Sci. Instrum. 75, 5119 (2004).

22. Stoner, R. J. \& Maris, H. J. Kapitza conductance and heat flow between solids at temperatures from 50 to 300 K. Phys. Rev. B 48 , 16373 (1993)

23. Monachon, C., Weber, L. \& Dames, C. Thermal Boundary Conductance: A Materails Science Perspective. Annu. Rev. Mater. Res. 46, 433-63 (2016).

24. Eichfeld, S. M. et al. Highly Scalable, Atomically Thin WSe ${ }_{2}$ Grown via Metal-Organic Chemical Vapor Deposition. ACS Nano 9 , 2080-2087 (2015).

25. Liu, L. C., Ge, M., Ma, Y., Abbas, A. N. \& Zhou, C. Step-Edge-Guided Nucleation and Growth of Aligned WSe ${ }_{2}$ on Sapphire via a Layer-over-Layer Growth Mode. ACS Nano 9, 8368-8375 (2015).

26. Lee, J.-H. et al. Reliable Piezoelectricity in Bilayer WSe ${ }_{2}$ for Piezoelectric Nanogenerators. Adv. Mater. 29, 1606667 (2017)

27. Tao, L. et al. Experimental and theoretical evidence for the ferromagnetic edge in WSe $\mathrm{H}_{2}$ nanosheets. Nanoscale 9, 4898 (2017).

28. Bozheyev, F., Harbauer, K., Zahn, C., Friedrich, D. \& Ellmer, K. Highly (001)-textured p-type WSe ${ }_{2}$ Thin Films as Efficient LargeArea Photocathodes for Solar Hydrogen Evolution. Sci. Rep. 7, 16003 (2017).

29. Smyth, C. M., Addou, R., McDonnell, S., Hinkle, C. L. \& Wallace, R. M. WSe ${ }_{2}$-contact metal interface chemistry and band alignment under high vacuum and ultra high vacuum deposition conditions. 2D Mater. 4, 025084 (2017).

30. Franken, P. A., Hill, A. E., Peters, C. W. \& Weinreich, G. Generation of Optical Harmonics. Phys. Rev. Lett. 7, 118 (1961).

31. Kim, C.-J. et al. Stacking Order Dependent Second Harmonic Generation and Topological Defects in h-BN Bilayers. Nano. Lett. 13, 5660-5665 (2013).

32. Li, Y. et al. Probing Symmetry Properties of Few-Layer $\mathrm{MoS}_{2}$ and h-BN by Optical Second-Harmonic Generation. Nano Lett. 13, 3329-3333 (2013).

33. Hsu, W.-T. et al. Second Harmonic Generation from Artificially Stacked Transition Metal Dichalcogenide Twisted Bilayers. ACS Nano 8, 2951-2958 (2014).

34. Zeng, H. et al. Optical signature of symmetry variations and spin-valley coupling in atomically thin tungsten dichalcogenides. Sci. Rep. 3, 1608 (2013).

35. Thomsen, C., Maris, H. J. \& Tauc, J. Picosecond acoustics as a non-destructive tool for the characterization of very thin films. Thin Solid Films 152, 217-223 (1987).

36. Hsieh, W.-P. \& Cahill, D. G. Ta and $\mathrm{Au}(\mathrm{Pd})$ alloy metal film transducers for time-domain thermoreflectance at high pressures. J. Appl. Phys. 109, 113520 (2011).

37. Wei, C., Zheng, X., Cahill, D. G. \& Zhao, J.-C. Invited Article: Micron resolution spatially resolved measurement of heat capacity using dual-frequency time-domain thermoreflectance. Rev. Sci. Instrum. 84, 071301 (2013). 
38. Feser., J. P. \& Cahill, D. G. Probing anisotropic heat transport using time-domain thermoreflectance with offset laser spots. Rev. Sci. Instrum. 83, 104901 (2012).

39. Kang, K., Koh, Y. K., Chiritescu, C., Zheng, X. \& Cahill, D. G. Two-tint pump-probe measurements using a femtosecond laser oscillator and sharpedged optical filters. Rev. Sci. Instrum. 79, 144901 (2008).

\section{Acknowledgements}

This work was supported in part by the Science Research Center and the Basic Science Research Program through the National Research Foundation of Korea (NRF) funded by the Ministry of Science, ICT \& Future Planning (Nos 2015R1A5A1009962, 2018R1A2B2005331). This work was supported by the "GRI (GIST Research Institute)" Project through a grant provided by GIST in 2018.

\section{Author Contributions}

Y.-G.C. and J.S.L. designed the experiments together with D.-G.J. and H.I.J. T.Y.K. and S.-W.K. fabricated the bare WSe $\mathrm{W}_{2}$ samples, and Y.-G.C. and H.I.J. deposited metal films on them. Y.-G.C., D.-G.J. and H.I.J. performed time-domain thermoreflectance measurements. C.J.R. and Y.-G.C. conducted the second-harmonic generation measurement. G.K. and B.S.M. conducted x-ray photoemission measurements. All authors discussed the results. Y.-G.C. and J.S.L. wrote the manuscript. J.S.L. supervised the project.

\section{Additional Information}

Supplementary information accompanies this paper at https://doi.org/10.1038/s41598-019-44091-9.

Competing Interests: The authors declare no competing interests.

Publisher's note: Springer Nature remains neutral with regard to jurisdictional claims in published maps and institutional affiliations.

(c) (i) Open Access This article is licensed under a Creative Commons Attribution 4.0 International License, which permits use, sharing, adaptation, distribution and reproduction in any medium or format, as long as you give appropriate credit to the original author(s) and the source, provide a link to the Creative Commons license, and indicate if changes were made. The images or other third party material in this article are included in the article's Creative Commons license, unless indicated otherwise in a credit line to the material. If material is not included in the article's Creative Commons license and your intended use is not permitted by statutory regulation or exceeds the permitted use, you will need to obtain permission directly from the copyright holder. To view a copy of this license, visit http://creativecommons.org/licenses/by/4.0/.

(c) The Author(s) 2019 